\section{Brains, cytokines and nightmares}

\section{Carlos Martínez-A}

Neuroimmunomodulation. Editors S. M. McCann and J. M. Lipton. Karger. 6/yr. USA \$514, elsewhere SFr668 (institutional); USA \$257, elsewhere SFr334 (personal).

WHAT is the meaning of a name? I guess that in searching for a title, the editors arrived at the answer Don Quixote gave to Sancho Panza: "it means what you understand of it". Thus Neuroimmunomodulation seeks to provide a home for new developments in a panoply of related disciplines covering the immunological, endocrinological and neurological sciences. The rapid increase in knowledge in these areas has been at the molecular level. But cellular and systemic perspectives are likely to be important in the future and might well become the principal areas of interest for this new journal.

Each issue aims to carry reviews as well as original articles. But the journal seems to be drifting towards becoming a reviewsoriented publication. As with many new periodicals, the quality of the papers is variable. Many of the contributors have direct links with members of the editorial board, which might explain the rapid manuscript acceptance speed. The journal is well produced, although the figures and tables look slightly old-fashioned. Unfortunately, $\mathrm{Neu}$ roimmunomodulation does not yet allow authors to submit manuscripts by e-mail.

The issues reviewed concentrate mainly on cytokines and neuroendocrine control of inflammation. Given the limited success of many of the competing journals already in these areas, the future of Neuroimmunomodulation is not guaranteed unless it carves out a distinct identity.

But the more trendy the papers it accepts, the more likely it is to succeed. If it is to speak to immunologists, endocrinologists, neurobiologists and physicians in the same language and to break down the barriers that have long separated cellular- and molecular-oriented science in these fields, some improvements are still needed. Although the niche for such a journal might seem obvious, this is a challenging goal and I wish the editors the best of luck.

Let me end by quoting Cervantes again. In Don Quixote de la Mancha, Don Quixote tells Sancho Panza that it is a tradition among masters to promote their servants to governors in the kingdoms they conquer. But he points out that "the adventure of becoming governor of one of these kingdoms, although potentially of interest, is an adventure that in most of the cases ends in nightmare". I hope not on this occasion.

Carlos Martínez-A is in the Department of Immunology and Oncology, CSIC Centro Nacional de Biotecnología, Universidad Autónoma, Campus de Cantoblanco, 28049 Madrid, Spain.

\title{
Superdrug or superbug?
}

\section{Curtis Gemmell}

\section{Microbial Drug Resistance: Mecha- nisms, Epidemiology, and Disease. Editor Alexander Tomasz. Liebert. 4/yr. \$156.}

THE threat that bacteria would defeat the best efforts of the pharmaceutical industry and become untreatable has been expounded over the years by many authors. It is no coincidence that the advent of this journal follows the appearance of multiple-drug-resistant Mycobacterium tuberculosis, especially among AIDS patients, and of epidemic methicillin-resistant Staphylococcus aureus as a hospital infection.

Medical microbiology in particular has witnessed a proliferation of specialist journals in recent years. Inevitably, there is a temporary withdrawal of support from well-established journals by authors attracted to the editorial board of a newcomer. Here board members contribute five out of eleven papers in the first issue, two out of thirteen in the third, and one out of nine in the fourth. Unfortunately, the journal has had to resort to running the proceedings of a commercially sponsored symposium early in its history (the second issue). In the first issue, only five full papers and one brief case report constitute original previously unpublished findings; the third issue, though, contains a few more case reports as well as three reviews (two short and one long). Only by the fourth issue are there truly original papers, so there does seem to be a market, albeit fragile, for this journal.

There is also likely to be strong competition with two other well-established journals (Antimicrobial Agents and Chemo-therapy and Journal of Antimicrobial Chemotherapy). It seems that many research groups are waiting in the wings to see whether the journal will establish its own niche. So my verdict at this stage (only possible in Scottish law) is "not proven".

Curtis Gemmell is in the Department of Bacteriology, Medical School, University of Glasgow, Glasgow G4 OST, UK.

\section{Translation manual}

\author{
Fran Balkwill
}

Cytokines and Molecular Therapy. Editors M. Talpaz and F. Herrmann. Dunitz. 4/yr. USA \$195, Europe £125, elsewhere $£ 130$ (institutional); USA $\$ 99$, elsewhere $£ 65$ (personal).

NATURE readers may think that 'translational research' has something to do with RNA and proteins, but a 'translational' scientist of the 1990 s is more likely to be grappling with the tortuous transition of research from bench to bedside. An increasing interest in 'translating' advances in molecular biology to clinical problems such as cancer and autoimmunity has predictably led to a rash of new journals focusing on cytokine therapy, therapeutic immunology, gene therapy or a combination of all three. Cytokines and Molecular Therapy belongs to the latter category with its stated aim of "disseminating leading-edge research in the major fields of cytokines and molecular therapy".

Its main areas of interest are haematology, oncology and immunology, but articles from other specialities are encouraged. These interests are reflected in the contents of the sample issues sent for review. Each contains two or three detailed reviews and three to five original research papers. The reviews generally provide excellent, extensive and up-to-date perspectives of areas such as cytokine modulation of neonatal haemopoiesis, molecular approaches to cancer immunotherapy, the molecular biology of Gaucher disease, and the involvement of the interleukin-1 system in leukaemia. The quality and relevance of the original papers are rather more variable, as might be expected from a journal in its infancy. Some sort of editorial comment would also be useful, especially to place original articles in a wider context.

The editors and editorial board comprise a galaxy of the best clinical and translational scientists, which should guarantee the success of this journal. But there are strong competitors, both new and old. It remains to be seen how many good and original papers this demanding field of research will generate, papers ideally of the standard found in Nature Medicine. If there are not enough original papers to sustain all of these journals, Cytokines and Molecular Therapy would perform a worthwhile function by concentrating on its comprehensive and useful reviews.

Fran Balkwill is at the Biological Therapies Laboratory, Imperial Cancer Research Fund, Lincoln's Inn Fields, London WC2A 3PX, UK. 